\title{
ANÁLISIS DE LOS EFECTOS DISPERSIVOS Y NO LINEALES EN UN CANAL ÓPTICO EMPLEANDO MÉTODOS NUMÉRICOS
}

\author{
Arturo Peralta Sevilla ${ }^{1, *}$, Milton Tipán Simbaña ${ }^{2}$ y Ferney Amaya Fernández ${ }^{3}$
}

\section{Resumen}

En este documento, presentamos el modelado de un canal de fibra óptica mediante la resolución de la Ecuación No Lineal de Schrödinger (NLSE). Se presentan las dos formas de solución para la NLSE: la forma analítica y la forma numérica empleando el método SSF (Split-Step Fourier Transform). En la simulación se consideran efectos lineales como la dispersión cromática y los efectos no lineales. Uno de los efectos no lineal es el efecto Kerr, del que se derivan los efectos de auto modulación fase (Self Phase Modulation, SPM) y modulación de fase cruzada (Cross Phase Modulation, XPM). Los métodos de solución son empleados para simular y visualizar los efectos de propagación a través de la fibra óptica. Se analizan los efectos de propagación para un escenario de red de acceso óptica con fibra mono-modo estándar (Single Mode Fiber, SMF), con longitudes de fibra de 20 y $40 \mathrm{~km}$ y tasas de bits entre 1,25 y 100 Gbps. De otro lado, son presentados los fenómenos no lineales como dispersión estimulada de Raman (Stimulated Raman Scattering, SRS) y dispersión estimulada de Brillouin (Stimulated Brillouin Scattering, SBS). Se presentan las ecuaciones para modelar SRS. Se presentan resultados de simulación de la amplificación Raman en un escenario seleccionado.

Palabras clave: Ecuación No Lineal de Schrödinger, efecto Kerr, Método numérico Split-Step Fourier, amplificación Raman.

\section{Abstract}

In this document, we present the modeling of an optical channel solving the Non Linear Schrödinger Equation (NLSE). We present two alternatives to solve the NLSE: the analytical solution and the numerical solution with the Split-Step Fourier Transform method. In the simulation, we consider the linear effects, such as the chromatic dispersion, and the nonlinear effects. One of the nonlinear effects is the Kerr effect, responsible for other nonlinear effects such as the Self Phase Modulation (SPM) and Cross Phase Modulation (XPM). The solution methods are employed in this paper to simulate and visualize the propagation effects through the optical fiber. We select an scenario of an optical access network with a single-mode fiber with fiber lengths of 20 and $40 \mathrm{~km}$ and data bit rates from 1,25 to 100 Gbps. On the other hand, we also present the nonlinear effects Stimulated Raman Scattering (SRS) and Stimulated Brillouin Scattering (SBS). We present the equations to model the SRS effect. We present simulation results with Raman amplification in a selected scenario.

Keywords: Non Linear Schrödinger Equation, Kerr effect, Numerical method Split-Step Fourier, Raman amplification.

\footnotetext{
1,* Magíster en Gestión de Telecomunicaciones, estudiante del Doctorado en Ingenierías Área Telecomunicaciones de la Universidad Pontificia Bolivariana, Medellín, Colombia, es Docente - Investigador, Grupo GITEL, a tiempo completo en la Universidad Politécnica Salesiana, Cuenca, Ecuador. Autor para correspondencia aperaltas@ups.edu.ec

${ }^{2}$ Estudiante del Doctorado en Ingenierías Área Telecomunicaciones de la Universidad Pontificia Bolivariana, Medellín, Colombia, es Docente - Investigador, Grupo GIETEC, a tiempo completo en la Universidad Politécnica Salesiana, Quito, Ecuador.

${ }^{3} \mathrm{PhD}$. Ingeniería Área Telecomunicaciones, es Docente-Investigador, Grupo GIDATI, a Tiempo Completo en la Universidad Pontificia Bolivariana, Medellín, Colombia. Autor para correspondencia ferney.amaya@upb.edu.co

Recibido: 19-02-2014, Aprobado tras revisión: 18-03-2014.

Forma sugerida de citación: Peralta, A., Tipán, M. y Amaya, F. (2014). "Análisis de los efectos dispersivos y no lineales en un canal óptico empleando métodos numéricos". InGENIUS. N. ${ }^{\circ} 11$, (Enero-Junio). pp. 5-17. ISSN: 1390-650X.
} 


\section{Introducción}

En la última década se ha observado un importante despliegue de las redes ópticas en los diferentes segmentos de la infraestructura de telecomunicaciones. De esta forma, en el acceso se encuentra la tecnología PON (por sus siglas del inglés Passive Optical Network) que permite a los operadores ofrecer servicios de fibra hasta el hogar (Fiber to the home, FTTH). En [1], se proponen arquitecturas híbridas que emplean TDM y WDM (Multiplexación por división de tiempo con multiplexación por división en la longitud de onda) en la llamada arquitectura TDM-PON/WDM-PON, como alternativas de migración y escalamiento, procurando tener el mínimo costo en [2] y ofreciendo un gran ancho de banda comparado con las tecnologías actuales HFC (Hybrid Fiber Coaxial) y DSL (Digital Subscriber Line).

En las etapas de distribución y core se observa un incremento en las tasas de bits y número de longitudes de onda por fibra a través de tecnologías como DWDM (Dense Wavelength Division Multiplexing) y modulaciones avanzadas, en [3] se propone WDM-PON utilizando fuentes de luz mediante el uso de MachZehnder (MZ), alcanzando 10 Gbps en el enlace de bajada con OFDM (Optical Orthogonal Frequency Division Multiplexing) y 2.5 Gbps en el enlace de subida con OOK (On-Off Keying). En este caso se amplifica la señal mediante la tecnología SOA (Semiconductor Optical Amplifier) y la re modulación se realiza con un modulador externo. Por otro lado, en [4] se propone WDM-PON alcanzando hasta 13 Gbps.

El despliegue de la tecnología óptica obedece al enorme crecimiento en el consumo de ancho de banda de las aplicaciones fijas, móviles y de nuevos servicios y aplicaciones como las ciudades inteligentes y las redes inteligentes. En [5] se propone la arquitectura de una red óptica multiservicios con soporte para Smart Grid, en donde el trayecto de migración es a través del método de cascada WDM/TDM-PON, considerando flujos de datos para diferentes aplicaciones y con una proyección hacia 10 años. En este mismo sentido, en [6] se analiza la necesidad de poseer dos canales independientes, uno para el flujo de la información derivada de Smart Grid y otro para el flujo de información convencional de usuario final.

Por esta razón, se hace de vital importancia entender los fenómenos que limitan la máxima tasa de bits y alcance en la transmisión de información que viaja a través de la fibra óptica

$\mathrm{Al}$ respecto, varios trabajos se han realizado con el objetivo de describir, mediante simulaciones, los diferentes efectos de propagación a través de la fibra óptica. En [7] se plantea un método numérico mediante diferencias finitas donde también se considera el ruido blanco gaussiano, para resolver la NLSE, las simulaciones son realizadas con pasos de $100 \mathrm{~m}$. Esto permite analizar los efectos no lineales SPM (Self-Phase Modulation) y XPM (Cross-Phase Modulation). En [8] se analizan los diferentes efectos de propagación a través de la fibra para señales ópticas moduladas en fase codificadas en Retorno a Cero (RZ) y No Retorno a Cero (RNZ).

En [9] se obtienen experimentalmente resultados de la relación portadora a ruido CNR (Carrier to Noise Ratio) para canales analógicos de Frecuencia Modulada (FM) y de Televisión por Cable (CATV). En [10] se obtienen soluciones analíticas aproximadas a la NLSE para verificar los fenómenos SPM y SRS. En [11] los autores plantean la solución de la NLSE mediante el método de SSF y se analiza una forma de compensación empleando fibra compensadora DCF (Dispersion Compensating Fiber). En [12] se presenta un modelo en tiempo discreto sobre la base del método SSF y sus efectos de transmitir sobre un canal aditivo de ruido blanco gaussiano AWGN (Additive White Gaussian Noise), al modelo discreto se le añaden fuentes de luz y amplificadores de fibra dopada con erbio EDFA (Erbium Doped Fiber Amplifier).

En este artículo se presenta una síntesis de cómo llegar a obtener la Ecuación No Lineal de Schrödinger (NLSE) a partir de las ecuaciones de Maxwell - sección 2, en la sección 3 se resuelve la NLSE en forma analítica y numérica, dando en esta última un algoritmo de cómo ejecutar el método, en la sección 4 se plantea como verificar los efectos no lineales de dispersión estimulada de Raman (SRS) y dispersión estimulada de Brillouin (SBS), en la sección 5 se presentan los resultados y se discute la simulación a diferentes distancias y velocidades de datos, se finaliza comparando parámetros de desempeño; finalmente en la sección 6 presentamos las conclusiones.

\section{Modelo del canal óptico}

En esta sección se obtiene la NLSE a partir de las ecuaciones de Maxwell. La NLSE permite modelar y simular los efectos de propagación a través de la fibra óptica.

$\mathrm{Al}$ ser la fibra óptica un material dieléctrico, la densidad volumétrica de carga y la densidad superficial de corriente de conducción son cero $\left(\rho_{\mathbf{v}}=0, \overline{\mathbf{J}}_{\mathbf{e}}=0\right)$. Con estas consideraciones, las ecuaciones de Maxwell en forma diferencial en función de la coordenada temporal t $\mathbf{t}$ y de la coordenada espacial $\mathbf{r}$, para el campo eléctrico $\overline{\mathbf{E}}$ y para la intensidad de campo magnético $\overline{\mathbf{H}}$, propagándose a través de una fibra óptica se presenta en (1-4):

$$
\nabla \cdot \bar{H}=0
$$




$$
\begin{gathered}
\nabla \times \bar{E}=-\mu \frac{\partial \bar{H}}{\partial t} \\
\nabla \cdot \bar{E}=0 \\
\nabla \times \bar{H}=\frac{\partial \bar{D}}{\partial t}
\end{gathered}
$$

Donde $\mu \mathrm{y} \epsilon$ son la permeabilidad magnética y permitividad eléctrica de la fibra óptica respectivamente y $\bar{D}$ es el vector desplazamiento eléctrico que se expresa en función del campo eléctrico y del vector de polarización $\bar{P}$ de la siguiente forma:

$$
\bar{D}=\epsilon_{0} \bar{E}+\bar{P}
$$

Hallando el rotacional a ambos lados de la ecuación 2:

$$
\nabla \times(\nabla \times \bar{E})=-\mu \frac{\partial}{\partial t}(\nabla \times \bar{H})
$$

Empleando $\nabla \times(\nabla \times \bar{E})=\nabla(\nabla \cdot \bar{E})-\nabla^{2} \bar{E}$ y reemplazando 4 y 5 se llega a:

$$
\nabla^{2} \bar{E}=-\mu_{0} \frac{\partial^{2}}{\partial t^{2}}\left(\epsilon_{0} \bar{E}+\bar{P}\right)
$$

Puede expresarse el vector polarización $\overline{\mathbf{P}}$ en función de una componente lineal y en una componente no lineal de la siguiente forma:

$$
\bar{P}=\bar{P}_{L}+\bar{P}_{N L}
$$

Siendo la componente lineal definida como:

$$
\bar{P}=\epsilon_{0} \mathrm{x}_{\mathrm{e}}^{(1)} \bar{E}
$$

Y la componente no lineal del vector de polarización:

$$
\bar{P}_{N L}=\epsilon_{0} \epsilon_{\mathrm{NL}} \bar{E}
$$

Donde $\mathrm{x}_{\mathrm{e}}^{(1)}$ es la susceptibilidad eléctrica de primer orden y $\epsilon_{\mathrm{NL}}$ es la permitividad no lineal del material.

Reemplazando la expresión del vector de polarización en la ecuación 7 se obtiene:

$$
\nabla^{2} \bar{E}=-\mu_{0} \epsilon_{0}\left(1+\mathrm{x}_{\mathrm{e}}^{(1)}+\epsilon_{\mathrm{NL}}\right) \frac{\partial^{2} \bar{E}}{\partial t^{2}}
$$

Considerando que la permitividad relativa del material puede expresarse en términos de la susceptibilidad eléctrica de primer orden

$$
\epsilon_{r}=1+\mathrm{x}_{\mathrm{e}}^{(1)}
$$

y que la permitividad del material es la suma de las componentes lineal y no lineal

$$
\epsilon=\epsilon_{\mathrm{r}}+\epsilon_{\mathrm{NL}}
$$

y empleando la ecuación

$$
c=1 / \sqrt{\mu_{0} \epsilon_{0}}
$$

se obtiene la ecuación de onda para el campo eléctrico a través de una fibra óptica:

$$
\nabla^{2} \bar{E}=-\frac{\epsilon}{c^{2}} \frac{\partial^{2} \bar{E}}{\partial t^{2}}
$$

$\mathrm{Al}$ hallar la transformada de Fourier (se reemplaza $\partial / \partial t$ por i $\omega$ ) de la ecuación 12 se obtiene la ecuación de onda para el campo eléctrico en el dominio de la frecuencia:

$$
\nabla^{2} \widetilde{E}+\frac{\omega_{0}^{2}}{c^{2}} \widehat{\epsilon}(\omega) \widetilde{E}=0
$$

La ecuación 13 se resuelve empleando el método de separación de variables, asumiendo que la solución del campo eléctrico en el dominio del tiempo tiene la siguiente forma:

$$
\begin{gathered}
\widetilde{E}(\mathbf{r}, \omega)=\widetilde{A}_{x}(z, \omega) \widetilde{F}_{x}\left(\mathbf{r}_{\perp}, \omega\right) \mathrm{e}^{j \beta(\omega) z} \mathbf{x}+\cdots+ \\
+\cdots+\widetilde{A}_{y}(z, \omega) \widetilde{F}_{y}\left(\mathbf{r}_{\perp}, \omega\right) \mathrm{e}^{j \beta(\omega) z} \mathbf{y}+\cdots+ \\
+\cdots+\widetilde{A}_{z}(z, \omega) \widetilde{F}_{z}\left(\mathbf{r}_{\perp}, \omega\right) \mathrm{e}^{j \beta(\omega) z} \mathbf{z}
\end{gathered}
$$

En la ecuación 14, $\widetilde{F}_{z}\left(\mathbf{r}_{\perp}, \omega\right)$ representa la transformada de Fourier de la distribución espacial del campo del modo fundamental de la fibra, que se asume independiente de los efectos de propagación a través de la fibra. $\widetilde{A}(z, \omega)$ es la transformada de Fourier de la envolvente del campo y $\beta(\omega)$ es la constante de propagación. Se ha encontrado que $\widetilde{F}_{z}\left(\mathbf{r}_{\perp}, \omega\right)$ es mucho menor que las otras dos componentes y, además,

$$
\widetilde{F}_{x}\left(\mathbf{r}_{\perp}, \omega\right) \approx \widetilde{F}_{y}\left(\mathbf{r}_{\perp}, \omega\right)
$$

por lo tanto la ecuación del campo eléctrico puede asumirse linealmente polarizada y reducirse a:

$$
\widetilde{E}(\mathbf{r}, \omega)=\widetilde{A}(\mathbf{z}, \omega) \widetilde{F}_{z}\left(\mathbf{r}_{\perp}, \omega\right) \mathrm{e}^{-j \beta(\omega) z}
$$

Aplicando el método de separación de variables, se puede encontrar la solución a la envolvente del campo en el dominio de la frecuencia $\widetilde{A}(\mathbf{z}, \omega)$, que es presentada en la ecuación 16 :

$$
2 j \beta_{0} \frac{\partial \widetilde{A}}{\partial z}+\left(\beta^{2}-\beta_{0}^{2}\right) \widetilde{A}=0
$$

El término $\beta$ de la ecuación 16 puede expresarse en función de su componente lineal $\beta_{L}$, que se aproxima mediante series de Taylor, y en función de $\Delta \beta$ que representa una pequeña cantidad de cambio debido a la contribución no lineal, tal como se presenta en 17:

$$
\begin{aligned}
& \beta=\beta_{L}+\Delta \beta, \\
& \beta_{L}=\sum_{k=0}^{\infty} \frac{\beta_{k} \Delta \omega^{k}}{k !}, \\
& \Delta \beta=\gamma|\bar{A}|^{2}+j \frac{\alpha}{2}
\end{aligned}
$$


Reemplazando 17 en 16:

$$
\frac{\partial \widetilde{A}}{\partial z}-j\left(\sum_{k=0}^{\infty} \frac{\beta_{k} \Delta \omega^{k}}{k !}+\gamma|\bar{A}|^{2}+j \frac{\alpha}{2}\right) \widetilde{A}=0
$$

Tomando la transformada inversa de 18 para obtener la ecuación en el dominio del tiempo, que se obtiene realizando el reemplazo:

$$
\Delta \omega^{k}=j^{k} \frac{\partial^{k}}{\partial t^{k}}
$$

Sustituyendo 19 en 18 se llega a la representación de la NLSE en el dominio del tiempo:

$$
\frac{\partial A}{\partial z}-j \sum_{k=0}^{\infty} \frac{\beta_{k}}{k !} \frac{\partial^{k} A}{\partial t^{k}}-j \gamma|\bar{A}|^{2} A+\frac{\alpha}{2} A=0
$$

Para nuestro interés la NLSE presentada en 20 se simplifica considerando únicamente la atenuación, los coeficientes de dispersión de segundo y tercer orden y el efecto Kerr, obteniendo:

$$
\frac{\partial A}{\partial z}+\underbrace{\underbrace{\frac{\beta_{2}}{2} \frac{\partial^{2} A}{\partial t^{2}}}_{2^{\circ} \text { orden }}-\underbrace{\frac{\beta_{3}}{6} \frac{\partial^{3} A}{\partial t^{3}}}_{3^{\circ} \text { orden }}}_{\text {Efecto dispersión }}+\underbrace{\frac{\alpha}{2} A}_{\text {Atenuación }}=\underbrace{\text { Lineal }}_{\text {SPM }} \underbrace{\underbrace{j \gamma|\bar{A}|^{2} A}_{\text {No lineal }}}_{\text {Efecto Kerr }}
$$

Normalmente el último término del lado izquierdo se puede eliminar para solucionar la ecuación ya que solo afecta la amplitud de la potencia de acuerdo al coeficiente de atenuación $\alpha$ de la fibra óptica. De esta forma, al solucionar la NLSE, puede solo considerarse la dispersión cromática y las no linealidades.

\section{Solución de la NLSE}

La NLSE puede resolverse en forma analítica o en forma numérica. La solución analítica solo es posible si se soluciona la NLSE en forma independiente considerando solo los efectos lineales o solo las no linealidades. Si se consideran simultáneamente las linealidades y no linealidades, el único método de solución posible de la NLSE es numérico.

\subsection{Solución analítica considerando la dispersión cromática}

Considerando solo el efecto de la dispersión cromática, la NLSE se expresa de la siguiente forma:

$$
\frac{\partial A}{\partial z}=-j \frac{\beta_{2}}{2} \frac{\partial^{2} A(z, t)}{\partial t^{2}}+\frac{\beta_{3}}{6} \frac{\partial^{3} A(z, t)}{\partial t^{3}}
$$

Tomando la transformada de Fourier a 22 para resolverla en el dominio de la frecuencia se obtiene:

$$
\frac{\partial \widetilde{A}}{\partial z}=\left(j \frac{\beta_{2}}{2} \omega^{2}-j \frac{\beta_{3}}{6} \omega^{3}\right) \widetilde{A}
$$

Reorganizando e integrando:

$$
\left\{\begin{array}{l}
\int \frac{\partial \widetilde{A}}{\widetilde{A}}=j \int\left(\frac{\beta_{2}}{2} \omega^{2}-\frac{\beta_{3}}{6} \omega^{3}\right) \partial z \\
\ln |\widetilde{A}|=j\left(\frac{\beta_{2}}{2} \omega^{2}-\frac{\beta_{3}}{6} \omega^{3}\right) z+C
\end{array}\right.
$$

Despejando $\widetilde{A}$ de 24 :

$$
\left\{\begin{array}{l}
\widetilde{A}(z, \omega)=\mathrm{e}^{j\left(\frac{\beta_{2}}{2} \omega^{2}-\frac{\beta_{3}}{6} \omega^{3}\right) z+C} \\
\widetilde{A}(z, \omega)=\mathrm{e}^{j\left(\frac{\beta_{2}}{2} \omega^{2}-\frac{\beta_{3}}{6} \omega^{3}\right) z} \mathrm{e}^{C} \\
K=\mathrm{e}^{C} \\
\widetilde{A}(z, \omega)=\mathrm{e}^{j\left(\frac{\beta_{2}}{2} \omega^{2}-\frac{\beta_{3}}{6} \omega^{3}\right) z} K
\end{array}\right.
$$

Considerando la condición inicial $\widetilde{A}(0, \omega)$ se puede determinar el valor para $\mathrm{K}$ :

$$
\{\widetilde{A}(0, \omega)=K
$$

Así la solución de la envolvente del campo en el dominio de la frecuencia es:

$$
\widetilde{A}(z, \omega)=\widetilde{A}(0, \omega) \mathrm{e}^{j\left(\frac{\beta_{2}}{2} \omega^{2}-\frac{\beta_{3}}{6} \omega^{3}\right) z}
$$

De 27 puede hallarse la función de transferencia de la fibra óptica en el dominio de la frecuencia considerando solo la dispersión cromática, que se expresa en la ecuación 28:

$$
H(\omega)=\frac{\widetilde{A}(z, \omega)}{\widetilde{A}(0, \omega)}=\mathrm{e}^{j\left(\frac{\beta_{2}}{2} \omega^{2}-\frac{\beta_{3}}{6} \omega^{3}\right) z}
$$

\subsection{Solución analítica considerando el efecto Kerr}

Considerando solo el efecto Kerr, la NLSE a partir de la ecuación 21 se expresa como:

$$
\frac{\partial A(z, t)}{\partial z}=-j \gamma|\bar{A}|^{2} A
$$

La envolvente del campo se sustituye con la siguiente expresión y restricciones:

$$
\left\{\begin{array}{l}
A=V \mathrm{e}^{\left(j \Phi_{\mathrm{NL}}\right)} \\
\frac{\partial V}{\partial z}=0 \\
\Phi_{\mathrm{NL}}=V^{2} \frac{L_{\mathrm{eff}}}{L_{\mathrm{NL}}}
\end{array}\right.
$$

Sustituyendo en 29 las ecuaciones de 30 da como resultado la solución para las no linealidades:

$$
A(L, T)=A(0, T) \mathrm{e}^{\left(j \frac{L_{\text {eff }}}{L_{\mathrm{NL}}}|A(0, T)|^{2}\right)}
$$

Efecto Kerr $\rightarrow L_{\text {eff }} \sim L_{\mathrm{NL}}$ 


\subsection{Solución numérica de la NLSE}

Para solucionar la NLSE presentada en la ecuación 21, considerando simultáneamente los efectos lineales y no lineales deben emplearse métodos numéricos y uno de los empleados es el SSF [11]. El método SSF asume que en una longitud infinitesimal $\Delta z$ de la de fibra óptica los efectos dispersivos y las no linealidades actúan en forma independiente. De esta forma la NLSE puede expresarse de la siguiente forma.

$$
\frac{\partial A(z, t)}{\partial z}=(\hat{D}+\hat{N}) A(z, t)
$$

Donde:

$$
\begin{array}{ll}
A(z, t): & \text { Amplitud de la envolvente del campo } \\
\hat{D}: & \text { Operador lineal } \\
\hat{N} & \text { Operador no lineal }
\end{array}
$$

Se han desarrollado variantes del método SSF y las más conocidas son el método simple, el completo y el simétrico, siendo este último el más utilizado por SSF. Se obtiene menor error con el simétrico, luego con el completo, siendo el simple el que presenta mayor error. La complejidad computacional del método simétrico es mayor que la de los otros métodos.

\subsubsection{Método simple}

Para este método los operadores lineal y no lineal se definen como se presenta en la ecuación 33:

$$
\left\{\begin{array}{l}
\hat{D}=j\left(\frac{\beta_{2}}{2} \omega^{2}-\frac{\beta_{3}}{6} \omega^{3}\right) \\
\hat{N}=j \gamma|\bar{A}|^{2}
\end{array}\right.
$$

De acuerdo al método simple primero se calcula, en el dominio de la frecuencia, la señal $A_{\mathrm{D}}(z+\Delta z, t)$ que resulta de considerar solo los efectos lineales en el intervalo $\Delta z$. Posteriormente, se calcula en el dominio del tiempo el efecto no lineal en el mismo intervalo $\Delta z$, siendo $A_{\mathrm{D}}(z+\Delta z, t)$ la señal a de entrada y $A_{\mathrm{D}}(z+\Delta z, t)$ la señal de salida. Esto se representa en la Figura 1.

\subsubsection{Método completo}

La definición de los operadores lineal y no lineal se mantiene como en 33, el algoritmo es similar al método simple con la diferencia que el cálculo de la NLSE en $\Delta z$ se realiza en tres pasos: primero se calcula el efecto de las linealidades en el intervalo $\Delta z / 2$, posteriormente se calcula el efecto de las no linealidades en el intervalo $\Delta z \mathrm{y}$, finalmente, se calcula el efecto de las linealidades en el intervalo $\Delta z / 2$, como se presenta en la Figura 2.

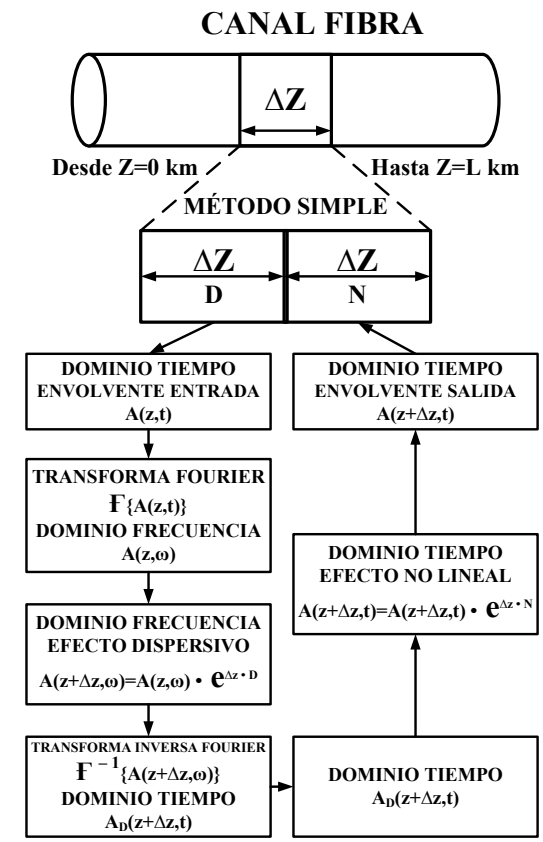

Figura 1. Algoritmo método simple.

CANAL FIBRA

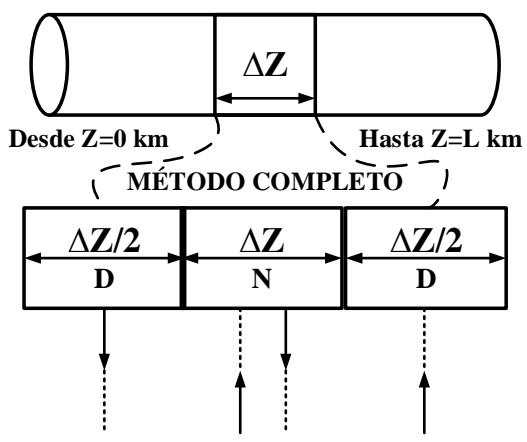

Figura 2. Algoritmo método completo.

\subsubsection{Método simétrico}

$\mathrm{El}$ algoritmo se ejecuta igual que para el método completo, la variante radica en cómo se representa el operador no lineal que se define como se presenta en la ecuación 34:

$$
\bar{N}=\mathrm{e}^{\int_{z}^{z+\Delta z} \bar{N}(z) d z} \approx[\bar{N}(z)+\bar{N}(z+\Delta z)] \frac{\Delta z}{2}
$$

\section{Efectos SRS y SBS}

Los efectos no lineales SRS y SBS no son representados en la NLSE. Estos efectos son el resultado de transferir la energía de una onda de frecuencia más alta (o longitud de onda menor) llamada señal de bombeo, a una de frecuencia más baja (o longitud de onda mayor) llamada señal de Stokes. En el caso de SBS la señal de 
Stokes se genera en sentido contra-propagante respecto a la señal de bombeo. El efecto SBS puede minimizarse limitando la máxima potencia de transmisión óptica.

En SRS la señal de Stokes puede ser co o contrapropagante. La máxima transferencia de potencia del bombeo a la señal de Stokes ocurre cuando las dos longitudes de onda tienen una diferencia cercana a $110 \mathrm{~nm}$, que es donde ocurre la máxima transferencia de potencia en SRS [13], [14], [15] para fibras ópticas de silicio. El efecto SRS es un efecto interferente entre dos longitudes de onda con espaciamientos cercanos a $110 \mathrm{~nm}$. El efecto SRS se emplea en la amplificación Raman si la señal de bombeo es continua (sin información). En el efecto SRS se genera ruido por emisión espontánea (Amplified Spontaneous Emission, ASE) que afecta el desempeño de los enlaces ópticos.

Para la simulación de la amplificación óptica Raman se emplean un conjunto de ecuaciones diferenciales ordinarias (ODE) acopladas como se describe en 35 :

$$
\left\{\begin{array}{l}
\frac{d P_{s}}{d z}=-\alpha_{s} P_{s} \pm P_{s} \sum_{i=1}^{N} \frac{g_{i}}{\Gamma A_{\mathrm{eff}}} P_{B \_i} \\
\frac{d P_{\mathrm{ASE}}}{d z}=-\alpha_{s} P_{\mathrm{ASE}} \pm \sum_{i=1}^{N} g_{i} P_{B \_i}\left(P_{\mathrm{ASE}}+2 h \nu \Delta \nu F_{i}\right)
\end{array}\right.
$$

Donde:

$P_{s}$ : Potencia de la señal

$P_{B_{i}}$ : Potencia i-ésimo bombeo

$P_{\mathrm{ASE}}$ : Ruido por emisión estimulada amplificada

$\alpha_{s}: \quad$ Constante de atenuación de la fibra

$g_{i}$ : Coeficiente de ganancia de Raman o Brillouin

$A_{\text {eff }}$ : Área efectiva

$h$ : $\quad$ Constante de Planck

$\nu$ : $\quad$ Frecuencia

$\Delta \nu: \quad$ Ancho de banda del ruido

$\Gamma: \quad$ Factor de polarización relativa

$F_{i}$ : Figura de ruido i-ésimo bombeo

El método numérico que se sugiere para resolver el sistema ODE es Runge Kutta de orden cuarto.

\section{Resultados de simulación de diferentes enlaces ópticos en el acceso}

En esta sección se presentan los resultados de simulación en un escenario de acceso óptico con diferente longitud y tasa de bits, para analizar los efectos de la dispersión y las no linealidades.

Para realizar las simulaciones se conformó un tren de 10 pulsos gaussianos como definen en [7] 36.

$$
A(T)=\sum_{k=1}^{N} a_{k} B\left(T-k T_{b}\right)
$$

Donde:

$a_{k}$ : Secuencia de bits

$T_{b}$ : Tiempo de bit

$B$ : k-ésimo pulso gaussiano

En la Tabla 1 se indican los valores de las constantes utilizadas en las simulaciones computacionales. Para todos los casos el medio de transmisión es una fibra mono-modo estándar (Single-Mode Fiber, SMF) tipo ITU-T G.652 y la frecuencia de operación es de $1550 \mathrm{~nm}$.

Tabla 1. Valores de las constantes.

\begin{tabular}{l|ll}
\hline$\lambda$ & 1550 & {$[\mathrm{~nm}]$} \\
$\beta_{2}$ & -21.668 & {$[\mathrm{ps} . \mathrm{ps} / \mathrm{km}]$} \\
$\mathrm{A}_{\text {eff }}$ & 80 & {$\left[\mathrm{um}^{2}\right]$} \\
$\alpha$ & 0.2 & {$[\mathrm{db} / \mathrm{km}]$} \\
$\gamma$ & 0.0013 & {$[1 /(\mathrm{mW} \mathrm{km})]$} \\
$\mathrm{h}$ & $6.6217 \mathrm{e}^{-34}$ & {$[\mathrm{~J} . \mathrm{s}]$} \\
\hline
\end{tabular}

Se generó una secuencia de bits aleatoria. La secuencia de bits y la señal óptica con los pulsos gaussianos se presentan en la Figura 3 y Figura 4.

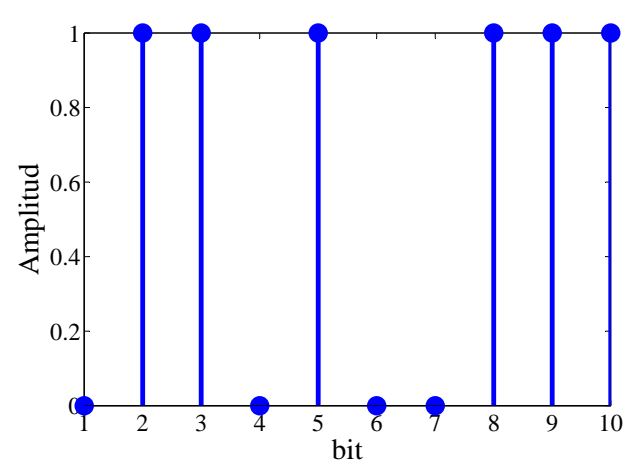

Figura 3. Secuencia de bits.

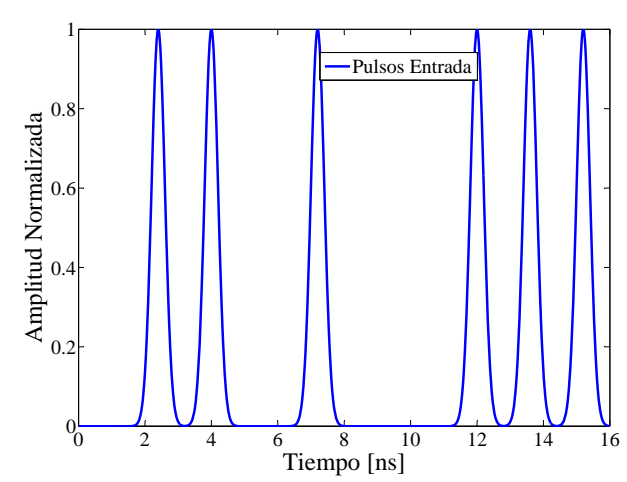

Figura 4. Tren de pulsos gaussianos iniciales.

Para el escenario de acceso óptico las simulaciones se realizaron para una sola longitud de onda, con longitudes de fibra óptica de 20 y $40 \mathrm{~km}$, a velocidades de $1.25,2.5,40$ y $100 \mathrm{~Gb} / \mathrm{s}$, tanto para la modulación por 
intensidad como para modulación por fase. Se calculan los parámetros de desempeño relacionados con el diagrama de ojo como son apertura del diagrama de ojo EC (Eye Closure), el factor Q y el BER (Bit Error Rate).

\subsection{Resultados para modulación óptica por intensidad}

Se analizan los resultados de simulación para una distancia de $20 \mathrm{~km}$ a tasas de bits de $1.25,40$ y $100 \mathrm{~Gb} / \mathrm{s}$. Por otro lado vale aclarar que los diagramas de ojo se construyeron sobre la base del número de puntos en la ventana que genera un pulso gaussiano; se adoptó un valor de 100 , por lo tanto para la elaboración del diagrama de ojo, se ha recortado a la mitad de cada pulso para visualizar mejor los efectos, o sea sobre una ventana de 50 puntos.

\subsubsection{Resultados con $\mathrm{z}=20 \mathrm{~km} \mathrm{y}$ $\mathrm{R}=1.25 \mathrm{~Gb} / \mathrm{s}$}

Como se aprecia en la Figura 5, la amplitud de la señal no sufre mayor variación debido a los efectos de dispersión, mientras que la fase presentada en la Figura 6 varía en magnitud debido a los efectos de propagación. Al comparar el diagrama de ojo de la señal de entrada a la fibra presentado en la Figura 7 con el de la salida de la Figura 8, prácticamente no se observan alteraciones.

\subsubsection{Resultados con $\mathrm{z}=20 \mathrm{~km}$ y $R=40 \mathrm{~Gb} / \mathrm{s}$}

Para una tasa de $\mathrm{R}=40 \mathrm{~Gb} / \mathrm{s}$ los efectos de propagación provocan una disminución en la amplitud y un desplazamiento de la señal, lo que puede observase en la Figura 9. Se aprecia un efecto similar al observado sobre la fase a $1.25 \mathrm{Gbps}$ como se presenta en la Figura 10. Esto se puede corroborar al comparar el diagrama de ojo a la entrada de la Figura 11 con respecto al de salida de la Figura 12, observándose una disminución del factor EC.

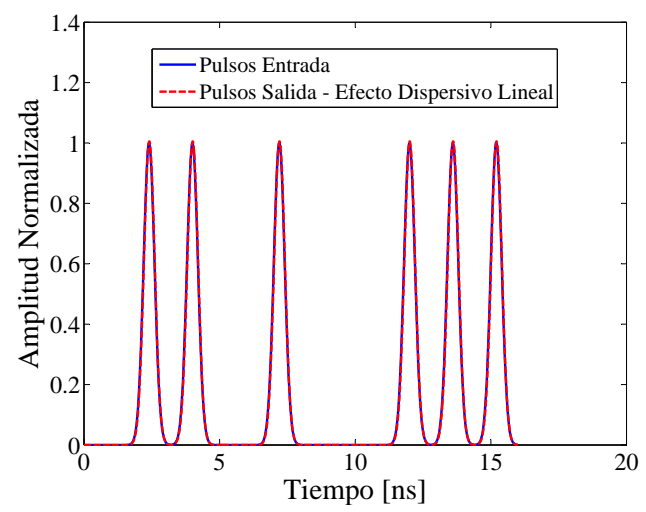

Figura 5. Amplitud Señal Entrada y Salida. $\mathrm{z}=20 \mathrm{~km}, \mathrm{R}=1.25 \mathrm{~Gb} / \mathrm{s}$, Modulación-IM.

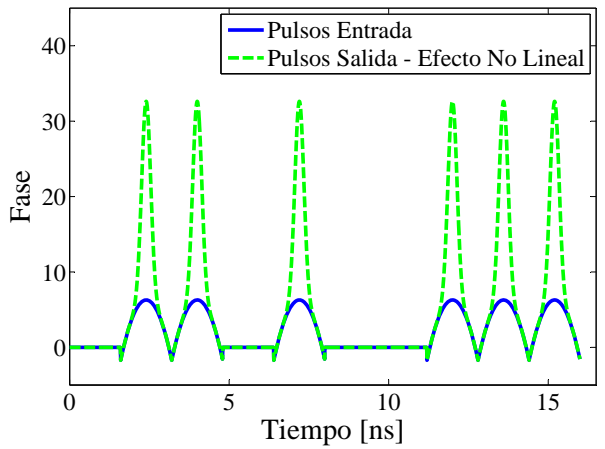

Figura 6. Fase Señal Entrada y Salida. $\mathrm{z}=20 \mathrm{~km}, \mathrm{R}=1.25 \mathrm{~Gb} / \mathrm{s}$, Modulación-IM.

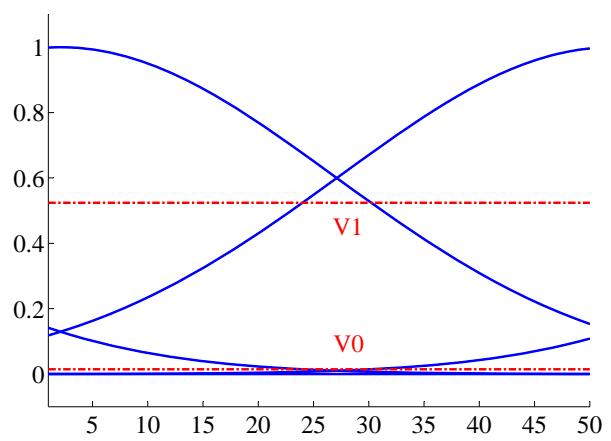

Figura 7. Diagrama de Ojo Señal Entrada. $\mathrm{z}=20 \mathrm{~km}, \mathrm{R}=1.25 \mathrm{~Gb} / \mathrm{s}$, Modulación-IM.

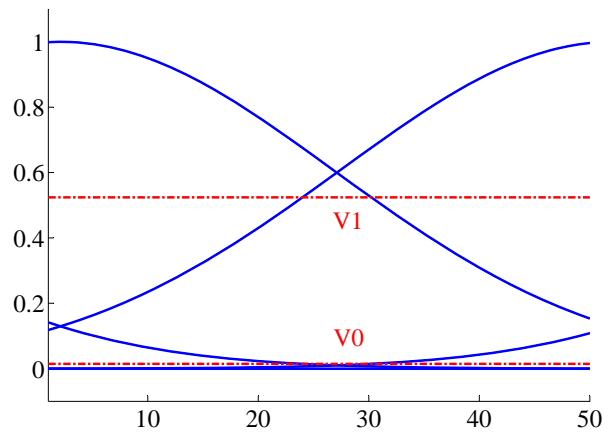

Figura 8. Diagrama de Ojo Señal de Salida. $\mathrm{z}=20 \mathrm{~km}, \mathrm{R}=1.25 \mathrm{~Gb} / \mathrm{s}$, Modulación-IM.

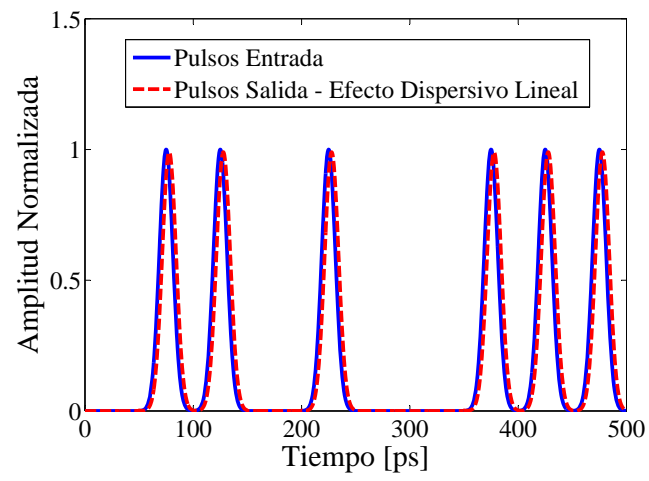

Figura 9. Amplitud Señal Entrada y Salida. $\mathrm{z}=20 \mathrm{~km}, \mathrm{R}=40 \mathrm{~Gb} / \mathrm{s}$, Modulación-IM. 


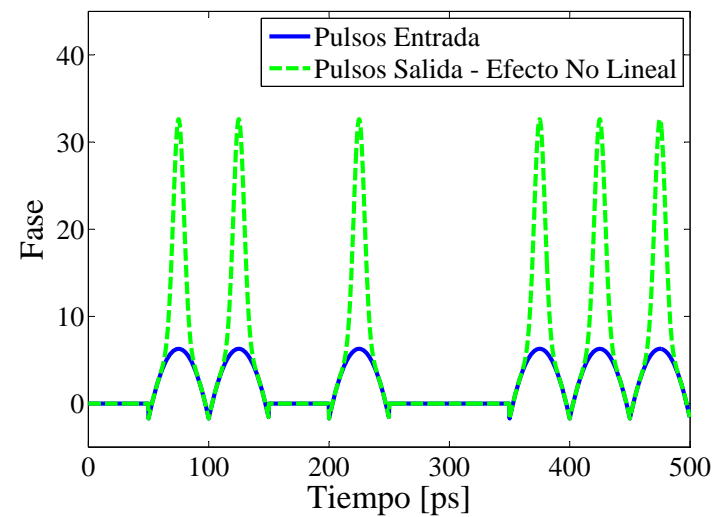

Figura 10. Fase Señal Entrada y Salida. $\mathrm{z}=20 \mathrm{~km}, \mathrm{R}=40 \mathrm{~Gb} / \mathrm{s}$, Modulación-IM.

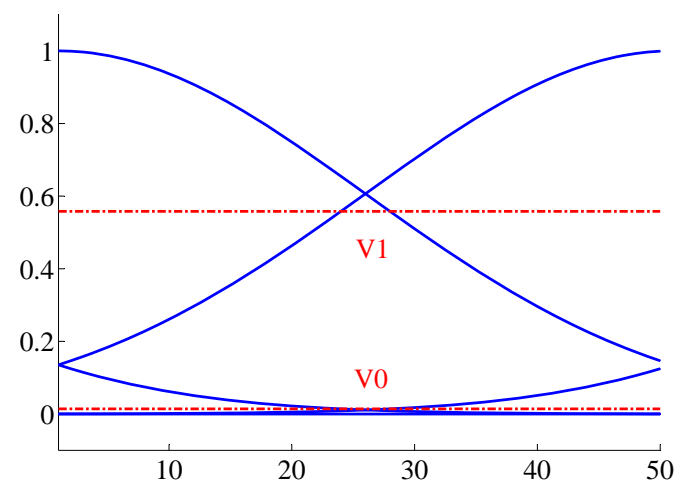

Figura 11. Diagrama de Ojo Señal Entrada. $\mathrm{z}=20 \mathrm{~km}, \mathrm{R}=40 \mathrm{~Gb} / \mathrm{s}$, Modulación-IM.

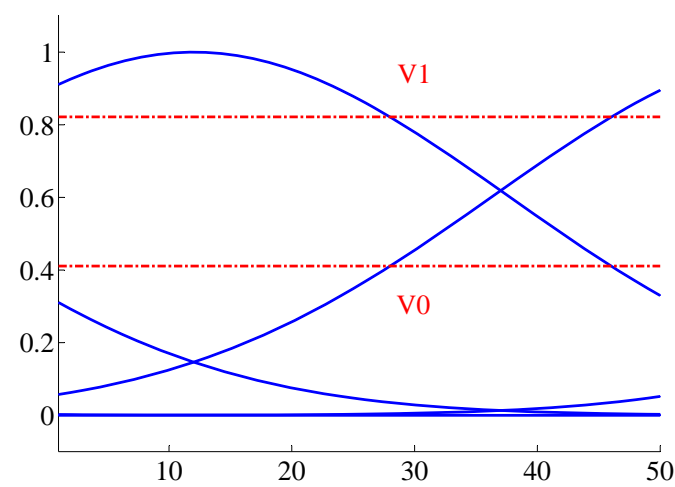

Figura 12. Diagrama de Ojo Señal de Salida. $\mathrm{z}=20 \mathrm{~km}, \mathrm{R}=40 \mathrm{~Gb} / \mathrm{s}$, Modulación-IM.

\subsubsection{Resultados con $\mathrm{z}=20 \mathrm{~km} \mathrm{y}$ $\mathrm{R}=100 \mathrm{~Gb} / \mathrm{s}$}

Los resultados presentados en la Figura 13 y Figura 14 para un tasa de $R=100 \mathrm{~Gb} / \mathrm{s}$ indican que la señal de salida es visiblemente afectada debido a los efectos dispersivos y no lineales. La amplitud de la señal disminuye casi un $50 \%$ y las alteraciones en la forma y ensanchamiento del pulso gaussino provocan una alta probabilidad de que se presente interferencia intersimbólica (intersymbol interference, ISI). Esto también se contrasta en el factor EC del diagrama de ojo que es muy pequeño, lo cual dificultaría la detección adecuada y reconocimiento entre un bit 1 y un bit 0 .

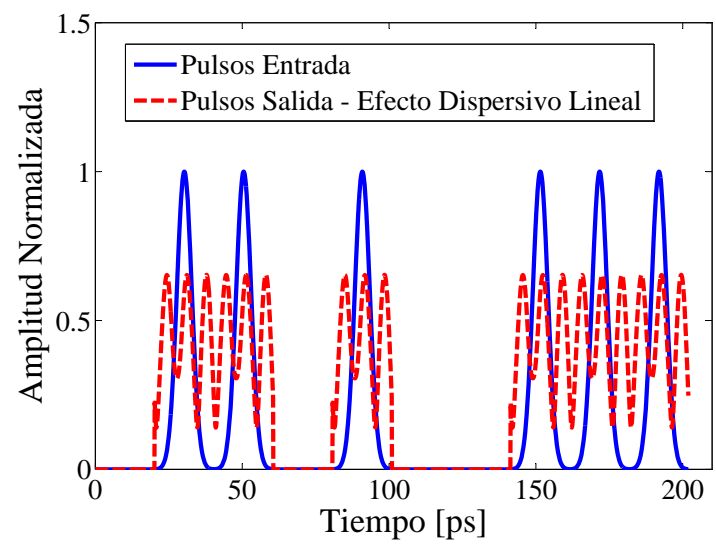

Figura 13. Amplitud Señal Entrada y Salida. $\mathrm{z}=20 \mathrm{~km}, \mathrm{R}=100 \mathrm{~Gb} / \mathrm{s}$, Modulación-IM.

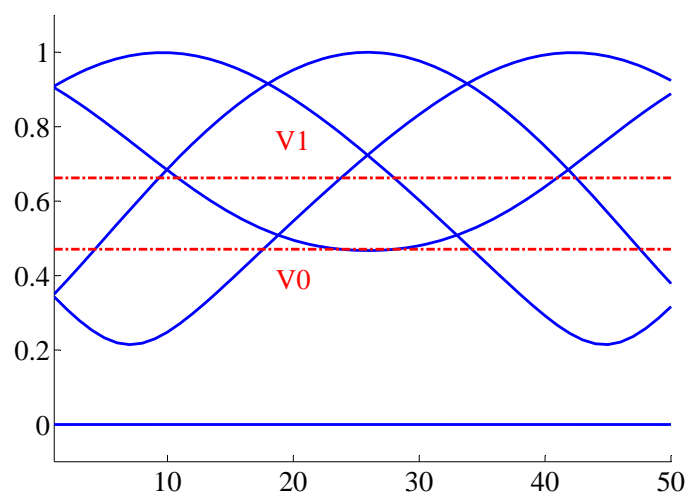

Figura 14. Diagrama de Ojo Señal de Salida. $\mathrm{z}=20 \mathrm{~km}, \mathrm{R}=100 \mathrm{~Gb} / \mathrm{s}$, Modulación-IM.

\subsection{Resultados para modulación óptica por fase}

En esta sección se analizan resultados de simulación con modulación óptica por fase. Para implementar esta modulación en casos reales se debe hacer mediante un modulador externo. A la señal se le adiciona ruido blanco gaussiano con una relación señal a ruido (Signal to Noise Ratio, SNR) de $40 \mathrm{~dB}$. La longitud de la fibra $\mathrm{z}=40 \mathrm{~km}$ y se simulan tasas de bits $\mathrm{R}=1.25 \mathrm{y}$ $40 \mathrm{~Gb} / \mathrm{s}$.

\subsubsection{Resultados con $\mathrm{z}=40 \mathrm{~km} \mathrm{y}$ $\mathrm{R}=1.25 \mathrm{~Gb} / \mathrm{s}$}

De lo mostrado en los resultados obtenidos con la modulación directa por intensidad la fase de la señal prácticamente se mantiene con las mismas alteraciones 
para todos los casos analizados esto se puede comprobar mirando la Figura 6 y la Figura 10, para el caso de la modulación por fase enviando los datos a distancias de $\mathrm{z}=40 \mathrm{~km}$, con una tasa de bits de $\mathrm{R}=1.25 \mathrm{~Gb} / \mathrm{s}$, e insertando ruido adicional $\mathrm{SNR}=40 \mathrm{~dB}$, se puede ver en la Figura 15 que el diagrama de amplitud de la señal de entrada sufre cambios leves debido a la dispersión y a las no linealidades, y más bien es un tanto más notorio el efecto del ruido sobre la señal de salida.

Esto también se puede comprobar mirando el diagrama de ojo para la señal de entrada en Figura 7 y compararlo con el diagrama de ojo para la señal de salida en la Figura 17. Los umbrales de reconocimiento para un cero lógico V0 y para el uno lógico V1 se encuentran dentro de la zona de máscara normal dada por el factor EC; la Figura 16 revela como el ruido y los efectos no lineales afectan severamente al diagrama de fase de la señal además debido al ruido introducido el Jitter se ve un tanto incrementado y esto provoca un incremento del BER.

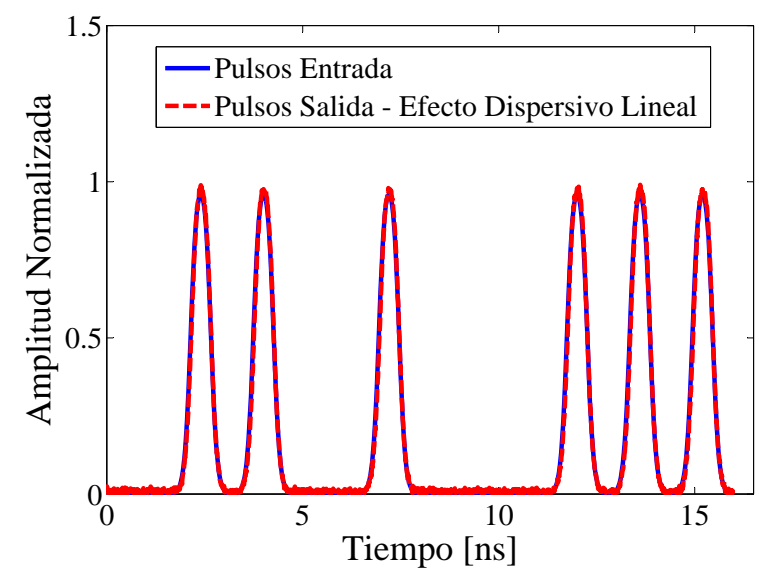

Figura 15. Amplitud Señal Entrada y Salida $\mathrm{z}=40 \mathrm{~km}$, $\mathrm{R}=1.25 \mathrm{~Gb} / \mathrm{s}$, Modulación-PM.

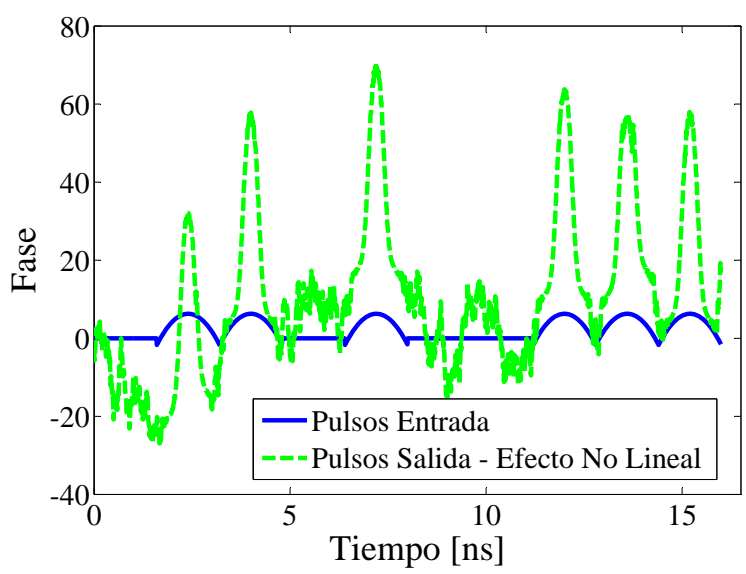

Figura 16. Fase Señal Entrada y Salida $\mathrm{z}=40 \mathrm{~km}$, $\mathrm{R}=1.25 \mathrm{~Gb} / \mathrm{s}$, Modulación-PM.

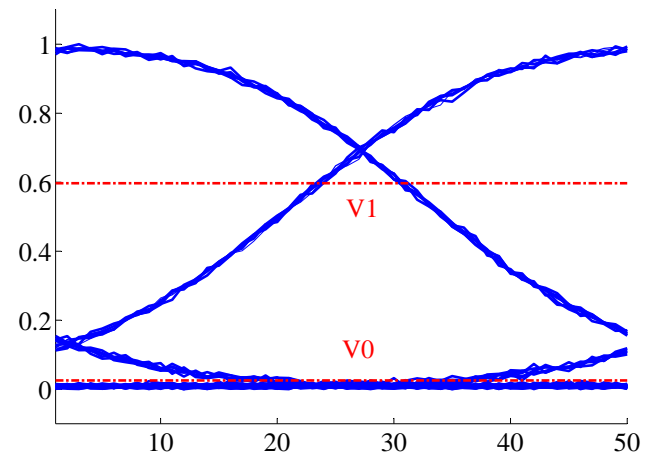

Figura 17. Diagrama de Ojo Señal de Salida z $=40 \mathrm{~km}$, $\mathrm{R}=1.25 \mathrm{~Gb} / \mathrm{s}$, Modulación-PM.

\subsubsection{Resultados con $\mathrm{z}=40 \mathrm{~km}$ y $R=40 \mathrm{~Gb} / \mathrm{s}$}

Cuando el tren de pulsos gaussianos es transmitido por el canal de fibra óptica modulados por fase, a una distancia de $40 \mathrm{~km}$ y a una tasa de bits de $\mathrm{R}=40 \mathrm{~Gb} / \mathrm{s}$, con SNR $=40 \mathrm{~dB}$, se observa en el diagrama de amplitud (Figura 18), que la señal de salida se ve ya afectada por los efectos de propagación que causan tres situaciones evidentes. Por un lado hay una atenuación en el valor de la amplitud, por otra parte hay un corrimiento de la señal hacia el lado derecho, y también se puede observar deformación en la constitución del pulso gaussiano. Esta deformación es más notoria cuando existen pulsos continuos en el tiempo. Estos efectos degradan el diagrama de ojo que se observa en la Figura 20, el cual se ve severamente afectado. Por otro lado en el diagrama de fase, Figura 19, la señal de salida también sufre notorias alteraciones.

\subsection{Evolución del pulso gaussiano}

Al resolver la NLSE por métodos numéricos puede observarse la evolución del pulso a lo largo de la fibra óptica. En la Figura 21 se observa la del pulso a través del tiempo y del espacio a través de una distancia $\mathrm{z}=40 \mathrm{~km}$ con una tasa de bits de $10 \mathrm{~Gb} / \mathrm{s}$, empleando modulación óptica por intensidad.

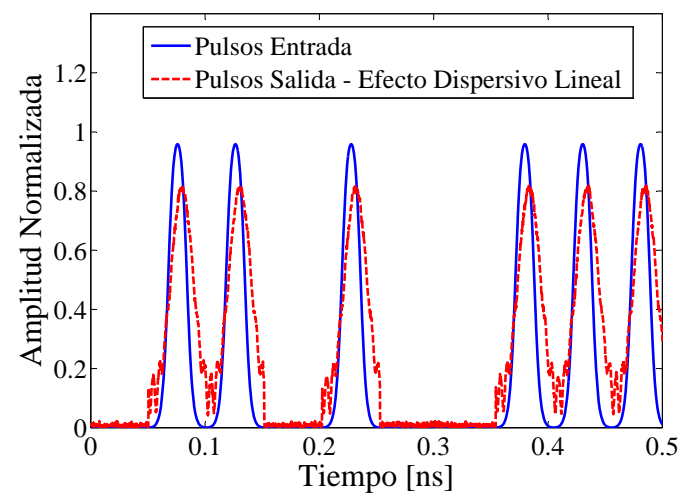

Figura 18. Amplitud Señal Entrada y Salida. $\mathrm{z}=40 \mathrm{~km}, \mathrm{R}=40 \mathrm{~Gb} / \mathrm{s}$, Modulación-PM. 


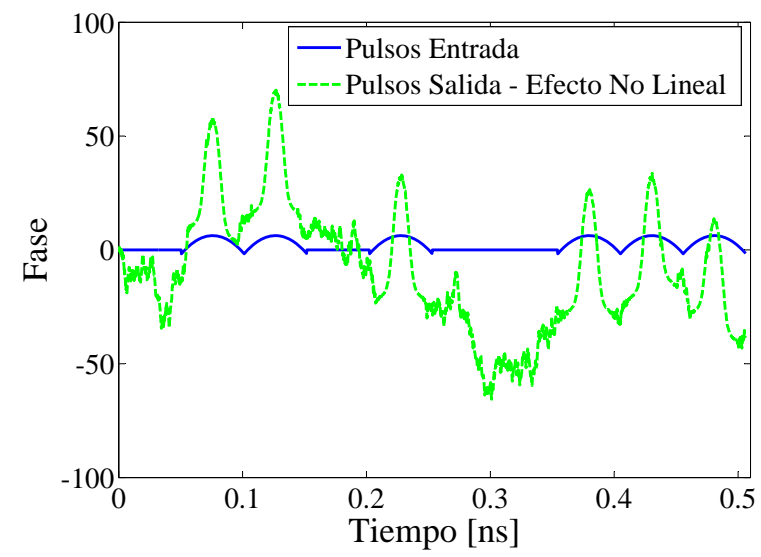

Figura 19. Fase Señal Entrada y Salida. $\mathrm{z}=40 \mathrm{~km}, \mathrm{R}=40 \mathrm{~Gb} / \mathrm{s}$, Modulación-PM.

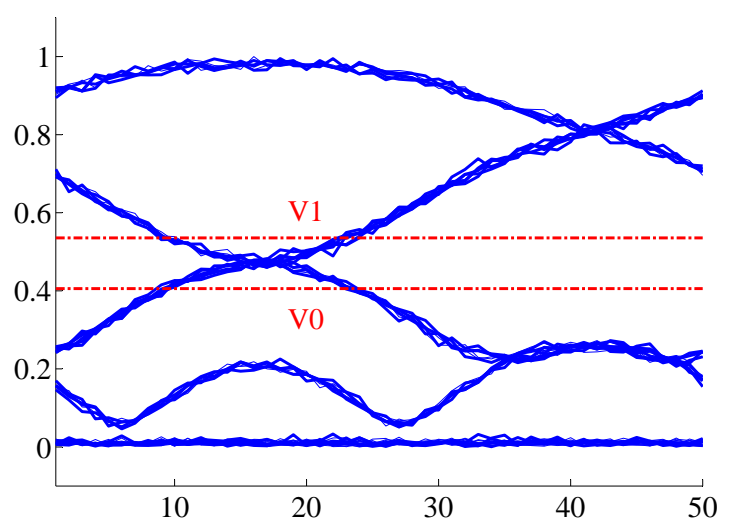

Figura 20. Diagrama de Ojo Señal de Salida. $\mathrm{z}=40 \mathrm{~km}, \mathrm{R}=40 \mathrm{~Gb} / \mathrm{s}$, Modulación-PM.

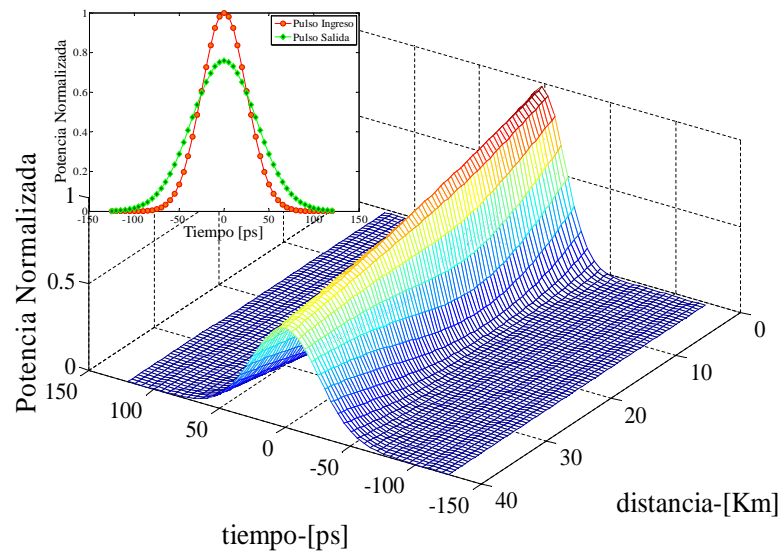

Figura 21. Evolución pulso gaussiano con SSF - simétrico.

\subsection{Comparación de parámetros de desempeño}

En este apartado se compara los parámetros de desempeño BER, Factor Q y Factor EC, como una función de la tasa de bits, las simulaciones se corrieron para $\mathrm{R}=\{1.25 ; 2.5 ; 40 ; 100\} \mathrm{Gb} / \mathrm{s}, \mathrm{y}$ considerando modulaciones directas por intensidad-IM, y modulaciones externas por fase-PM, para distancias de $20 \mathrm{y}$ $40 \mathrm{~km}$. Estos resultados son expuestos en la Figura 22, Figura 23 y Figura 24.

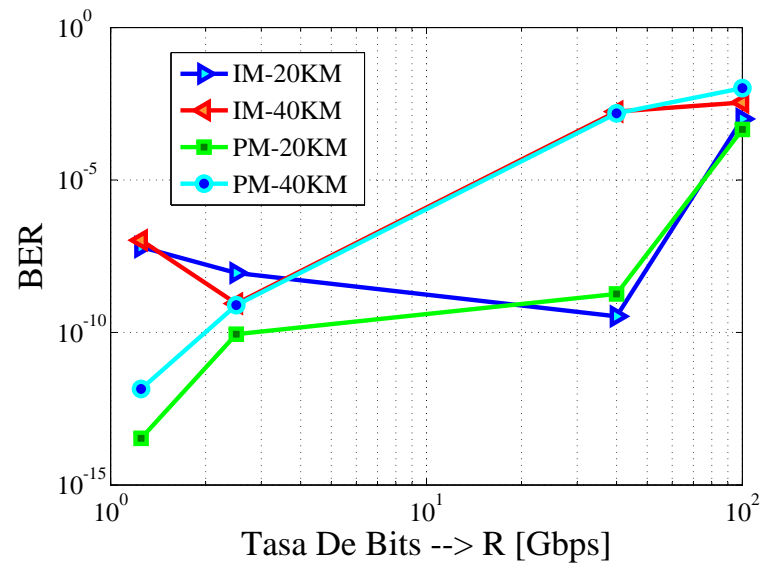

Figura 22. BER vs. $\mathrm{R}$ Con $\mathrm{z}=20 \mathrm{y} \mathrm{z}=40 \mathrm{~km}$.

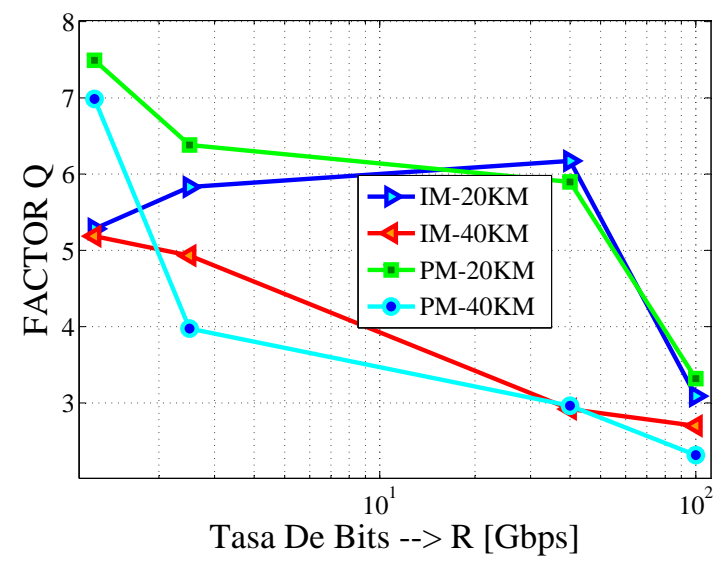

Figura 23. Q vs. $\mathrm{R}$ con $\mathrm{z}=20 \mathrm{y} \mathrm{z}=40 \mathrm{~km}$.

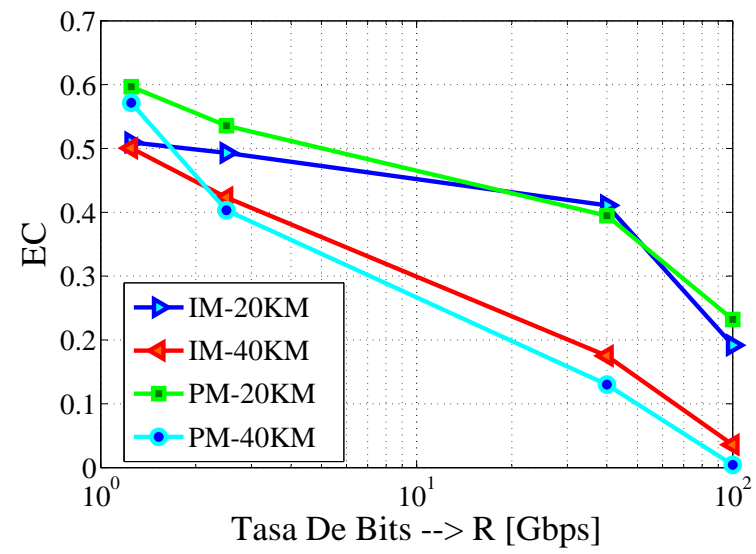

Figura 24. EC vs. $\mathrm{R}$ Con $\mathrm{z}=20 \mathrm{y} \mathrm{z}=40 \mathrm{~km}$. 


\subsection{Resultados de simulación para SRS}

Los resultados de la simulación del efecto SRS, sobre la base de las ecuaciones presentadas en 35 se han logrado mediante el escenario presentado en la Figura 25 que contiene un solo bombeo copropagante. Los resultados numéricos luego de procesar Runge Kutta de cuarto orden se exhiben en la Tabla 2 y Figura 26. Se observa trasferencia de potencia desde la señal de bombeo $\left(P_{b}\right)$ a la señal de entrada $\left(P_{s}\right)$ a medida que las señales se propagan por el medio de transmisión. Puede también observarse la evolución de la señal de ruido ASE.

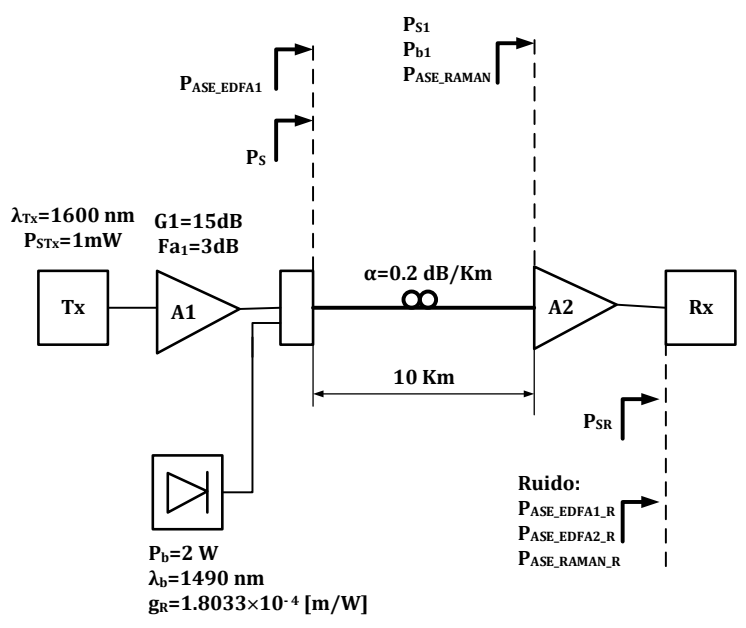

Figura 25. Escenario de enlace para simular SRS.

\section{Conclusiones}

Partiendo de las ecuaciones de Maxwell se ha demostrado un esquema de cómo llegar a obtener la NLSE, la cual permite simular y visualizar los fenómenos de propagación sobre un canal de fibra óptica. Se presentaron resultados de simulación considerando los efectos de la dispersión cromática y las no linealidades. Los resultados de simulación se obtuvieron solucionando la NLSE por métodos analíticos y numéricos.

Para la solución numérica de la NLSE se presentó el método SSF describiendo sus tres alternativas de solución. Una de las ventajas del uso del método numérico es la posibilidad de observar la evolución de un pulso a lo largo de la fibra en función de la potencia, el tiempo y la distancia.

Se obtuvieron resultados de simulación en forma gráfica que muestran tres parámetros de desempeño, como son BER, Factor Q, y Factor EC, como función de la tasa de bits $\mathrm{R}=\{1.25,2.5,40,100\} \mathrm{Gb} / \mathrm{s}$, donde se comparan pulsos modulados en forma directa por intensidad (IM) y pulsos modulados por fase para distancias de 20 y $40 \mathrm{~km}$. Pudo concluirse y verificarse que para bajas tasas de bits $\mathrm{R}<2.5$ Gbps la modulación PM presenta mejores características sea para las distancias simuladas. Sin embargo, a partir de tasas superiores a $\mathrm{R}>2.5 \mathrm{~Gb} / \mathrm{s}$, se nota una marcada diferencia entre los valores para un recorrido de $20 \mathrm{~km}$, con respecto a $40 \mathrm{~km}$. Por ejemplo, si se compara el BER para $40 \mathrm{~Gb} / \mathrm{s}$ con un recorrido de $20 \mathrm{~km}$, sea para IM o PM, se tiene $10^{-10}<\mathrm{BER}<10^{-8}$, mientras que para $40 \mathrm{~km}$, se tiene $10^{-4}<\mathrm{BER}<10^{-2}$.

En la Figura 27, se presenta una comparación mediante OOK (ON-OFF keying), para un longitud de onda de $\lambda=1567 \mathrm{~nm}$ con una tasa de transmisión $\mathrm{R}=10 \mathrm{Gbps}$, de resultados obtenidos mediante simulación y experimentales, algunos valores de experimentación se muestran en [16], las tendencias de las dos gráficas presentan un comportamiento muy similar lo cual indica que el modelo propuesto empleando los parámetros adecuados, serviría como una herramienta para simular escenarios previo a realizar montajes reales con una muy buena aproximación.

Finalmente se consiguieron simulaciones para verificar el comportamiento del efecto SRS con la utilización de una fuente de bombeo en sentido copropagante. Se concluyó que la potencia de la fuente de bombeo $\left(P_{b}\right)$, cede energía a la potencia de la señal $\left(P_{s}\right)$. Con esto se logra compensar los efectos debidos a pérdidas por atenuación. Sin embargo, la potencia del ruido tipo ASE, también se incrementa por el efecto de la amplificación óptica.

Para futuros trabajos se sugiere trabajar con varios canales ópticos en escenarios WDM.

También se sugiere realizar simulaciones con varias fuentes de bombeo en sentido co y contrapropagante.

Tabla 2. Resultados Runge Kutta.

\begin{tabular}{cccc}
\hline $\mathbf{z}$ & $\mathbf{P b}$ & $\mathbf{P s}$ & $\mathbf{P a s e}$ \\
\hline 0 & 2.000 .000 & 0.031630 & 0.00000000 \\
500 & 1.948 .454 & 0.036932 & 0.00066701 \\
1000 & 1.897 .271 & 0.042924 & 0.00142333 \\
1500 & 1.846 .373 & 0.049659 & 0.00227608 \\
2000 & 1.795 .687 & 0.057188 & 0.00323209 \\
2500 & 1.745 .141 & 0.065559 & 0.00429773 \\
3000 & 1.694 .672 & 0.074813 & 0.00547872 \\
3500 & 1.644 .221 & 0.084987 & 0.00677991 \\
4000 & 1.593 .742 & 0.096105 & 0.00820502 \\
4500 & 1.543 .198 & 0.108185 & 0.00975642 \\
5000 & 1.492 .566 & 0.121229 & 0.01143489 \\
5500 & 1.441 .836 & 0.135226 & 0.01323941 \\
6000 & 1.391 .015 & 0.150150 & 0.01516697 \\
6500 & 1.340 .127 & 0.165958 & 0.01721243 \\
7000 & 1.289 .213 & 0.182591 & 0.01936839 \\
7500 & 1.238 .332 & 0.199970 & 0.02162519 \\
8000 & 1.187 .561 & 0.218003 & 0.02397096 \\
8500 & 1.136 .992 & 0.236578 & 0.02639171 \\
9000 & 1.086 .734 & 0.255571 & 0.02887156 \\
9500 & 1.036 .906 & 0.274846 & 0.03139302 \\
10000 & 0.987637 & 0.294257 & 0.03393732 \\
\hline & & &
\end{tabular}




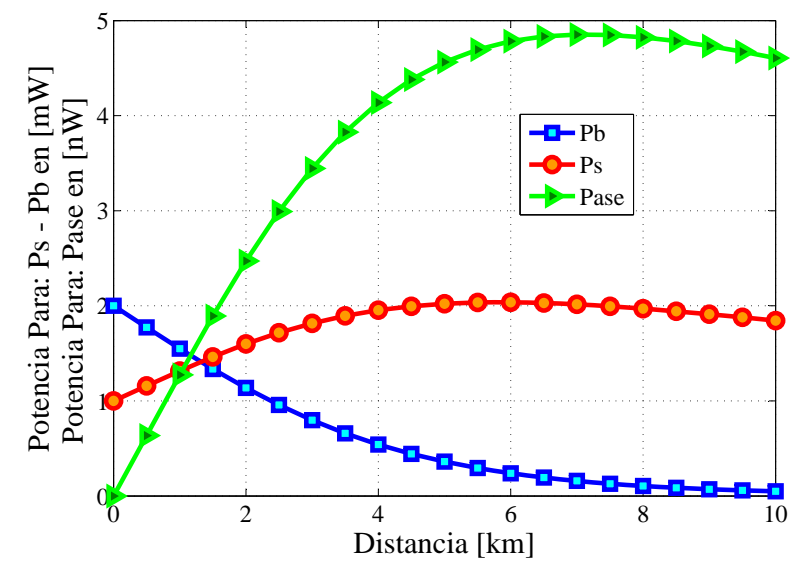

Figura 26. Transferencia de potencia con un bombeo efecto SRS.

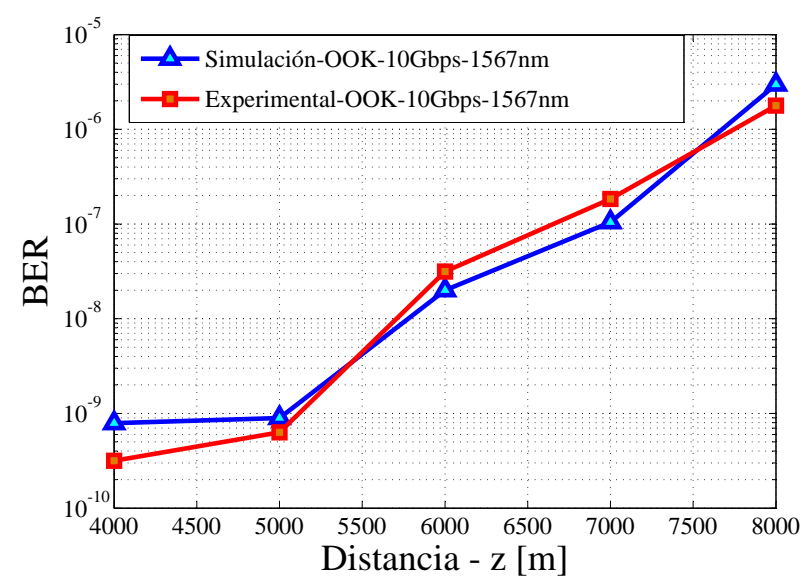

Figura 27. Comparación datos simulados y experimentales, con $\mathrm{R}=10 \mathrm{Gbps}, \lambda=1567 \mathrm{~nm}$, OOK.

\section{Agradecimientos}

Se agradece a la Universidad Pontificia Bolivariana, Medellín, Colombia y a la Universidad Politécnica Salesiana, sedes Cuenca y Quito, Ecuador, por la colaboración en cuanto a poner a disposición sus laboratorios de simulación para el desarrollo del presente trabajo.

\section{Referencias}

[1] M. S. Ahsan, M. S. Lee, S. Newaz, and S. Asif, "Migration to the next generation optical access networks using hybrid WDM/TDM-PON," Journal of Networks, vol. 6, no. 1, pp. 18-25, 2011.

[2] L. D. Truong, T. P. Do, N. T. Nguyen, and H. Q. Duong, "Impact of mesh topology in cost reduction of survivable hybrid WDM-TDM PON networks," in Proceedings of the Third Symposium on Information and Communication Technology. New York, USA: ACM, 2012, pp. 159-164.
[3] J. Huang, D. Liu, and C. Zeng, "10 Gb/s WDMPON using downstream OFDM and upstream OOK," Computer \& Information Science, vol. 4, no. 2 , p. $34,2011$.

[4] C. Chow, C. Yeh, Y. Wu, H. Chen, Y. Lin, J. Sung, Y. Liu, and C. L. Pan, "13 Gbit/s WDM-OFDM PON using RSOA-based colourless onu with seeding light source in local exchange," Electronics Letters, vol. 47, no. 22, pp. 1235-1236, 2011.

[5] A. Gómez-Martínez, F. Amaya-Fernández, R. Hincapie, J. Sierra, and I. Tafur-Monroy, "Optical access multiservice architecture with support to smart grid," in Communications and Computing (COLCOM), 2013 IEEE Colombian Conference on. IEEE, 2013, pp. 1-5.

[6] A. Peralta-Sevilla and F. Amaya-Fernández, "Evolución de las redes eléctricas hacia Smart Grid en países de la Región Andina," Revista Educación en Ingeniería, vol. 8, no. 15, pp. 48-61, 2013.

[7] X. Fu, J. Dai, J. Yu, and E. Yang, "Numerical simulation of multi-channel WDM transmission system in non-linear optical fiber communication system," in Electrical and Computer Engineering. Canadian Conference on, vol. 3. IEEE, 2004, pp. 1819-1822.

[8] F. Jacobs, J. Shaw, and V. Wongpaibool, "Effects of phase modulation and filtering on NRZ and RZ signals," in Signals, Systems and Computers. Conference Record of the Thirty-Fifth Asilomar Conference on, vol. 1. IEEE, 2001, pp. 13-17.

[9] R. Coenen and M. Jackson, "The impact of fiber dispersion on CNR in 80-channel wideband FM CATV transmission," in Nanostructures and Quantum Dots/WDM Components/VCSELs and Microcavaties/RF Photonics for CATV and HFC Systems, 1999 Digest of the LEOS Summer Topical Meetings. IEEE, 1999, pp. IV7-IV8.

[10] A. Ramprasad and M. Meenakshi, "A study on the propagation characteristics of pulses in optical fiber communication systems," in Signal Propagation on Interconnects, 2006. IEEE Workshop on. IEEE, 2006, pp. 263-266.

[11] M. Hamza and S. Tariq, "Split step fourier method based pulse propagation model for nonlinear fiber optics," in Electrical Engineering, 200\%. ICEE'0\%. International Conference on. IEEE, 2007, pp. $1-5$.

[12] L. Beygi, E. Agrell, P. Johannisson, M. Karlsson, and H. Wymeersch, "A discrete-time model for uncompensated single-channel fiber-optical links," Communications, IEEE Transactions on, vol. 60, no. 11, pp. 3440-3450, 2012. 
[13] I. D. Rukhlenko, M. Premaratne, and G. P. Agrawal, "Nonlinear silicon photonics: analytical tools," Selected Topics in Quantum Electronics, IEEE Journal of, vol. 16, no. 1, pp. 200-215, 2010.

[14] B. Neto, M. Rodrigues, E. Rocha, and P. Andre, "Stability analysis of raman propagation equations," in Transparent Optical Networks, 2009. ICTON'09. 11th International Conference on. IEEE, 2009, pp. 1-4.
[15] X. Liu, H. Zhang, and Y. Guo, "A novel method for raman amplifier propagation equations," Photonics Technology Letters, IEEE, vol. 15, no. 3, pp. 392-394, 2003.

[16] C. Xu, X. Liu, L. F. Mollenauer, and X. Wei, "Comparison of return-to-zero differential phaseshift keying and on-off keying in long-haul dispersion managed transmission," Photonics Technology Letters, IEEE, vol. 15, no. 4, pp. 617-619, 2003. 